\title{
An automatic multichannel generalized system for frequency measurement
}

\author{
G. Gomah ${ }^{\star}$ and A. Mostafa
}

National Institute for Standards (NIS), Time and Frequency Laboratory, Tersa St., El- Haram - P.O. Box: 136 Code No.12211, Giza, Egypt

Received: 20 November 2014 / Accepted: 14 January 2015

\begin{abstract}
Monitoring the performance of the primary frequency sources continuously through comparing it versus the transfer standards and the calibration of the secondary frequency sources periodically versus the primary ones are of the main missions assigned to any time and frequency laboratory either it was a calibration laboratory or a national metrological laboratory. An automatic Generalized System (GS) for monitoring/calibrating any frequency source that has a Relative Frequency Offset (RFO) greater than $1 \times 10^{-14} \mathrm{~Hz} / \mathrm{Hz}$ has been built. This GS is able to use either of two measurement methods according to the accuracy of the frequency source being measured. A graphical programming language, which is Labview, was used in writing the software required for both hardware control and data logging. So, the software can be easily reconfigured for any upgrading plans. Also a flexible arrangement for the hardware setup was used such that two measurement systems are merged in one system. So, according to the user needs the right hardware setup can be chosen. The results obtained by this GS were verified through comparing them to those generated by one of the commercial turnkey solutions.
\end{abstract}

Keywords: Generalized system; time interval measurement; phase comparison method; direct frequency measurement method; calibration system

\section{Introduction}

The importance of precise oscillators comes from that various technologies and applications rely on precise time and frequency sources. financial markets, wired and wireless telephone networks, radio and television broadcast stations, the electrical power grid, and the satellites systems are applications that rely on precise frequency sources. So frequency measurements have a special importance [1].

Precise or standard oscillators are categorized into two categories; mechanical oscillators and atomic oscillators. Quartz is a mechanical oscillator while rubidium, cesium, and hydrogen Maser are atomic oscillators. Quartz oscillators are the base for all the atomic oscillators [2]. In general to use an oscillator in a certain application we have to match the application requirements to the oscillator's specifications. Unfortunately, the manufacturer's specifications are not enough because the most important parameters of an oscillator, which are the frequency offset and frequency stability, are changing with time due to several reasons like aging, power outage, and change in the environmental conditions. Moreover, in case of failure, atomic oscillator works as Quartz oscillator that has a very different performance $[3,4]$. If this was the case for the standard frequency sources, what about the commercial ones? So all types of frequency sources have to be either continuously monitored (for primary standards) or periodically calibrated (for secondary standards) [1].

* Correspondence: gihan_gomah@yahoo.com
Moreover, monitoring the performance of the primary frequency sources is one of the main missions assigned to any time and frequency laboratory either it was a calibration lab or a national metrological lab Actually, any time and frequency calibration laboratory should maintain three automatic measurement systems. The first one is dedicated for monitoring the performance of its primary frequency sources (on a $24 / 7$ basis) by comparing them to each other or to a transfer standard. The second system is dedicated for calibrating precise frequency sources versus the primary ones. Since calibrating the general frequency sources do not need the same measurement accuracy needed by the precise ones. So general frequency sources can be calibrated using a less accurate and less time consuming measurement method. So it will be better for the calibration lab to dedicate a third setup for the not precise frequency sources coming from the industry. The three systems have almost the same basic construction specially if all the measurements are done in the time domain [5].

Calibrating or monitoring a precise oscillator is a long and time consuming process especially when measuring the long term stability. So some of the National Metrology Institutes (NMIs) (like the National Institute of Standards and Technology (NIST); the NMI of American) and international companies around the world (like Symmetricom) sell a compact and turnkey solutions for calibrating or monitoring precise oscillators [6]. The role of the operator is just to connect both the reference oscillator and the 
Device Under Test (DUT) to the system then the results are generated automatically after a certain measurement period.

Although having a turnkey solution is the easiest and fastest way to solve the problem but it has also drawbacks. For example, the operator will be confined to the options offered by the supplier and will not be able to satisfy the increasing demands of the customers, especially if it was a calibration lab. Moreover, if any part of this compact system fails the operator will not be able to replace it himself/herself without the supplier' aid. Because it is very likely the part that can replaces the failed one is not compatible with either the failed hardware or the software package that controls the overall system in a harmonized manner. The problem will be more complicated if the failed part was obsolete. In this case the operator will replace the overall system with a new one.

In this article an automatic multichannel GS for frequency measurements was built. The system has flexible measurement methods a flexible hardware setup, and a flexible software program. By that way it can achieve most of the lab' or customer' needs and can be easily upgraded to follow the continuous development in the field of electronics. The results obtained by this GS was verified through comparing them to those generated using one of the turn key solutions after measuring the same frequency sources by both systems.

\section{The measurement methods and the measurement setups}

In general, the main parameters to be measured when calibrating/monitoring a frequency source are the relative frequency offset (RFO) and the frequency stability [1]. For a successful measurement, it is preferred that the reference should outperform the DUT by a stability ratio of about 10:1. If a smaller ratio is used the calibration will take a longer period to be sure that we measure the performance of the DUT [7]. The measurement of the frequency offset and frequency stability can be done in the time domain by either of two measurement methods; the Phase Comparison Method (PCM), sometimes called the time interval measurement method, or the Direct Frequency Measurement Method (DFMM) [8-11] The PCM is more accurate than the DFMM but it needs a longer measurement period. In general, the PCM are used in monitoring the performance of the precise frequency sources while the DFMM is usually used in calibrating the commercial or general frequency sources that have less accuracy.

\subsection{The phase comparison method (PCM)}

Theoretically, the PCM implies measuring the running phase difference between the reference and DUT at the points that have the highest slew rate, as shown in Figure 1, using a time interval counter (TIC) [6]. The slew rate value has a direct effect on both the Trigger Timing Jitter error and the Trigger Level Timing error contributed to the measured frequency value. According to equations (1) and (2), it is shown that as the slew rate increases as the error contributed by the Trigger Jitter

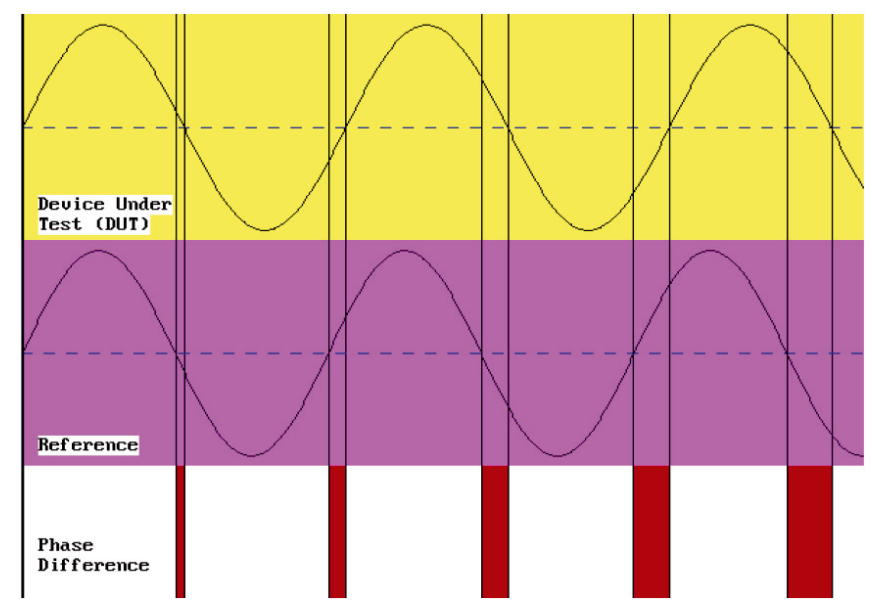

Fig. 1. Two sinusoidal signals with a changing phase relationship [6].

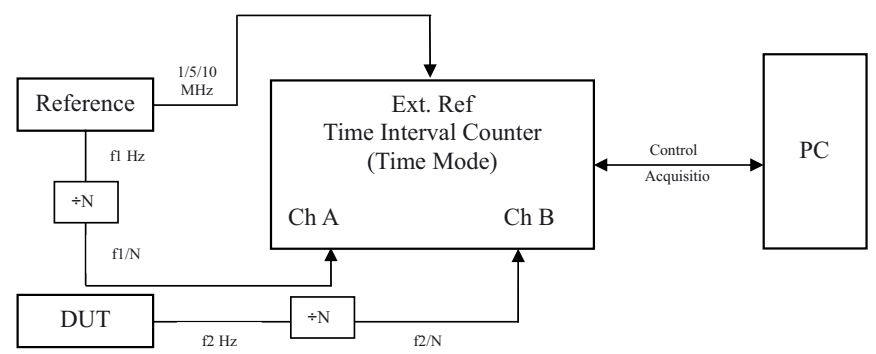

Fig. 2. The basic measurement setup for the phase comparison method.

and Trigger Timing Error decreases $[12,13]$. Practically and for sinusoidal signals, we use the optimum slew rate not the highest one [14]. Figure 2 shows a basic measurement setup for frequency measurement using the PCM

$$
\text { Trigger Timing Jitter }=\frac{\sqrt{\left(E_{\text {internal }}\right)^{2}+\left(E_{\text {signal }}\right)^{2}}}{\text { Input Slew Rate }}
$$

where $E_{\text {internal }}$ is the counter's internal noise and $E_{\text {signal }}$ is the input signal noise.

$$
\text { Trigger Level Timing Error }=\frac{15 \mathrm{mV}+0.5 \% \text { of setting }}{\text { Input Slew Rate }} .
$$

As shown in Figure 2, the frequency of both the reference and DUT is stepped down using frequency dividers before comparing their running phase difference. In this method it is recommended to use low frequency values, in the range of few $\mathrm{KHz}$, to avoid the rollover of the measured phase $[6,15]$. So, almost all the turnkey solutions use frequency dividers to step down the frequency.

Since pulsed signals have a higher slew rate than sine wave signals, it will be better to reshape the sinusoidal frequency of both the reference and DUT to a high slew rate pulses. This will help in decreasing the errors affecting the measured frequency value [16]. There are equipments that do both frequency division and signal reshaping; which are called Pulse Per Second (PPS) generators. The function of most of the PPS generators is to transform a frequency of $1 \mathrm{MHz}, 5 \mathrm{MHz}$, or $10 \mathrm{MHz}$ to a $1 \mathrm{~Hz}$ pulsed signal that has a very high slew rate and narrow pulse width [17]. Figure 3 


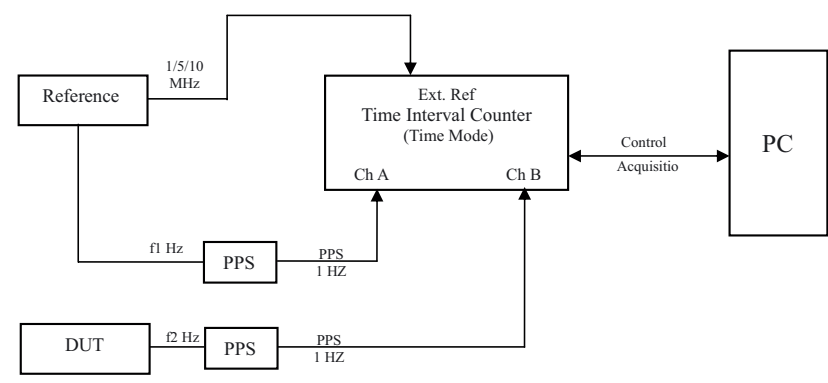

Fig. 3. The ideal measurement setup for the phase comparison method.

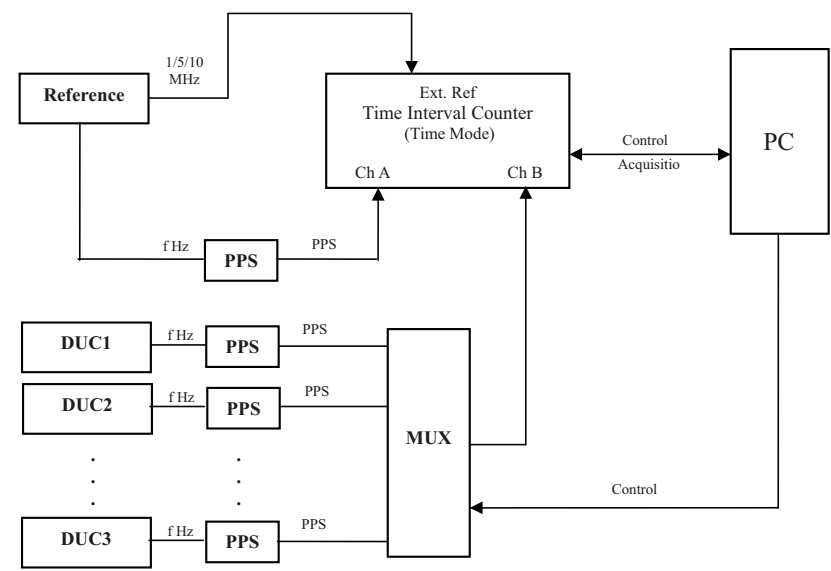

Fig. 4. A multichannel measurement system for the phase comparison method.

shows a more complicated single channel calibration setup that uses PPS generators.

When we use the PCM we use a TIC in the Time mode and use the DUT for opening one of the counter's gates and the reference for closing the other gate. To construct a multi channel calibration system that is able to measure many frequency sources at the same time using the same counter and the same reference, we have to connect the reference to one of the counter's gates and all the other frequency sources to the second gate. To rout multiple frequency sources to the same gate we have to use a multiplexer. The size of the multiplexer will determine the number of frequency sources (number of channels of the calibration system) that can be measured at the same time. Figure 4 shows a multichannel calibration system that uses the PCM.

This setup enables us from calibrating the $1 \mathrm{MHz}$, $5 \mathrm{MHz}$, or $10 \mathrm{MHz}$ frequency signals that are coming from any frequency source. This means also that any frequency source that has one of these frequency values within its operating range can be also calibrated using this setup.

\subsection{The direct frequency measurement method (DFMM)}

The frequency, $f$, of a repetitive signal is defined as the number of cycles of that signal per unit of time. It is represented by the following equation:

$$
f=n / t
$$

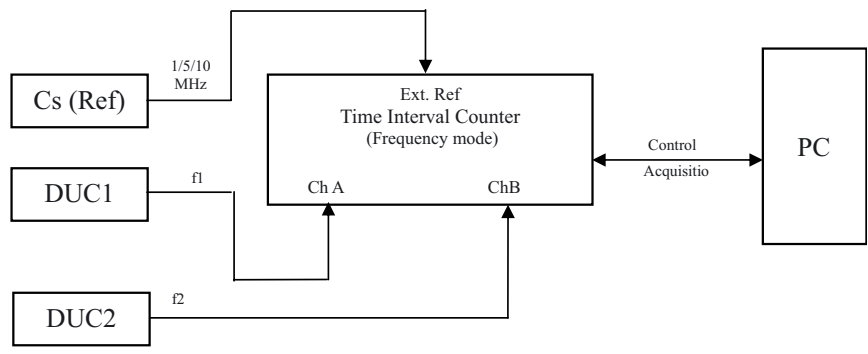

Fig. 5. Two channels measurement system for the direct frequency measurement method.

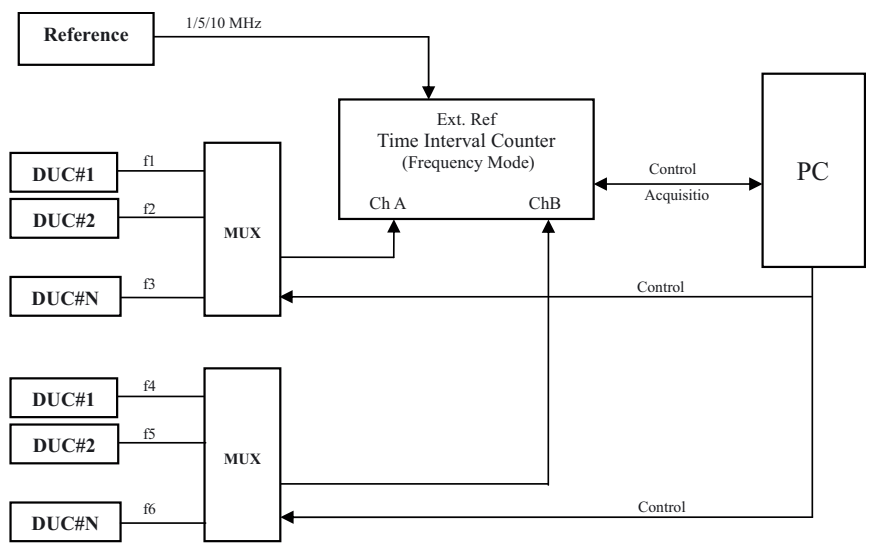

Fig. 6. Multi-channels measurement system for the direct frequency measurement method.

where $n$ is the number of cycles of the repetitive signal that occurs in a time interval, $t$. If $t=1 \mathrm{~s}$, then the frequency is expressed as $n$ cycles per second or $n$ Hertz [18]. According to equation (3), the frequency, $f$, of a repetitive signal is directly measured by a counter (in the Frequency mode) by counting the number of cycles, $n$, and dividing them by the measurement interval, $t$.

The measurement setup used for the DFMM is simpler than the one used for the PCM and this is the main advantage of this method. Figure 5 shows a two channel calibration system that uses the DFMM. On the other hand, the main disadvantage of the DFMM is that it has less measurement accuracy compared to the PCMM. To construct a multichannel calibration system a multiplexer should be added to route the multiple frequency sources to the same channel. For the DFMM, the number of channels is double the number that can be used using the PCMM. Figure 6 shows a multichannel calibration system for the DFMM.

Most of the turnkey calibration systems use an only one measurement method. But it is shown that the multichannel setup that uses the PCM can be easily turned into a multichannel DFMM system by inserting the PPS and changing the TIC mode. As shown in Figure 7, a switch can be added to enable inserting or not inserting the PPS to get an automatic multichannel GS capable of using two measurement methods for monitoring primary frequency sources and calibrating precise and general frequency sources. If the switch was an electronic switch, then it's operation can be controlled automatically 


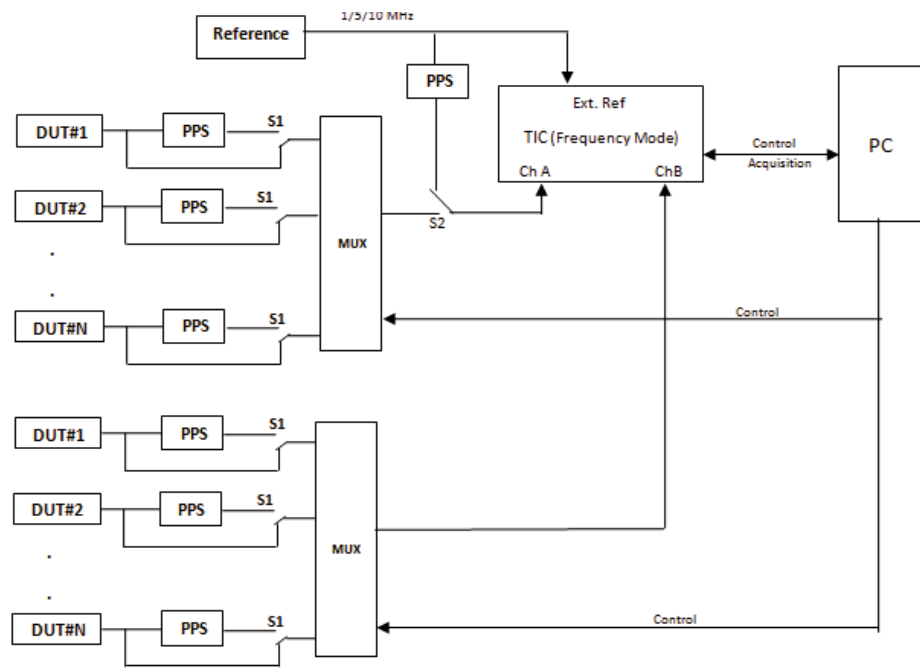

through the software. If it was a mechanical switch, then it's status will be adjusted manually before running the software. For a multichannel measurement setup that uses the PCM, S1 and S2 are connected to the PPS while the Time Interval Counter (TIC) operates in the Time mode. For a multichannel measurement setup that uses the DFMM; S1 is connected to the DUT, S2 is connected to the MUX, and the TIC operates in the Frequency mode. In all cases, the operating mode of the TIC will be controlled through the software. The multiplexer that was used in the GS enables us from calibrating up to 13 frequency sources at the same time.

\section{Software program for instrument control and data acquisition}

All the turn key calibration systems have the control and data acquisition software preinstalled and the user has not the right to have the source code. This type of software is usually written especially to control the instruments inside the system and according to the options offered by the manufacturer. Moreover, if an individual instrument in the setup fails, the user can't replace it by a similar one, from the point of view of hardware, because it will not work with the preinstalled software. The sole solution is replacing the failed instrument by the manufacturer. If the failed part was obsolete (this is a usual case), then the whole system should be replaced by a new one.

The functions of the software of such turnkey measurement systems include instruments control and data acquisition. In general, the instrument control is used in controlling all the electronic equipments in the measurement setup. In the case of the GS, instrument control include controlling the TIC's measurement parameters, the multiplexer's channels, and the electronic switches. For establishing a flexible program it is recommended to use a graphical programming language instead of the text based languages. Today, the graphical programming languages have almost the same capabilities of the text based programming languages with an increased flexibility [19].

The graphical programming language that was used with the GS was Labview from National Instruments.
Fig. 7. A multi-channel generalized system for frequency measurement.

For a full control on the equipments, the rule of thumb is to rebuild the hardware front panels of all the instruments that need control using the software. Figure 8 shows that the front panel of the TIC was fully transferred to the software. By that way, all the parameters of the TIC can be controlled easily through software. The counter used with the measurement system was the Universal Time Interval Counter SR620 from Stanford Research Systems.

Fortunately, the instrument control facility in Labview can control GPIB, serial, USB, Ethernet, PXI, or VXI instruments. This means that if we want to replace a failed instrument with another one that has a different model and different bus interface there will be no problem. This advantage can be used in continuously upgrading the system using the up to date TIC whatever the bus interface it is using.

\section{Verifying the generalized system (GS)}

To verify the results obtained by the GS they are compared to the ones that were generated by one of the turnkey solutions. At the Time and Frequency lab of NISEgypt there is already an American turnkey measurement system that was manufactured by NIST. This system is a five channels measurement system and is called Frequency Measurement and Analysis System (FMAS). The main function of this system is to measure the RFO and frequency stability of the standard frequency sources that have a RFO better than $1 \times 10^{-5} \mathrm{~Hz} / \mathrm{Hz}$ versus the primary standard of the lab. Moreover, it enables monitoring the performance of the lab' primary standards versus a transfer standard (like GPS disciplined oscillator). This system uses an only one measurement method; which is the PCM.

According to the used TIC, the GS can measure the frequency offset and frequency stability of all types of frequency sources, even those having a frequency offset worse than $1 \times 10^{-5} \mathrm{~Hz} / \mathrm{Hz}$.

To verify the results obtained by the GS, a rubidium $(\mathrm{Rb})$ standard frequency source is calibrated versus a cesium-based primary frequency standard, which is the cesium Beam Frequency Standard (5071 A high performance 


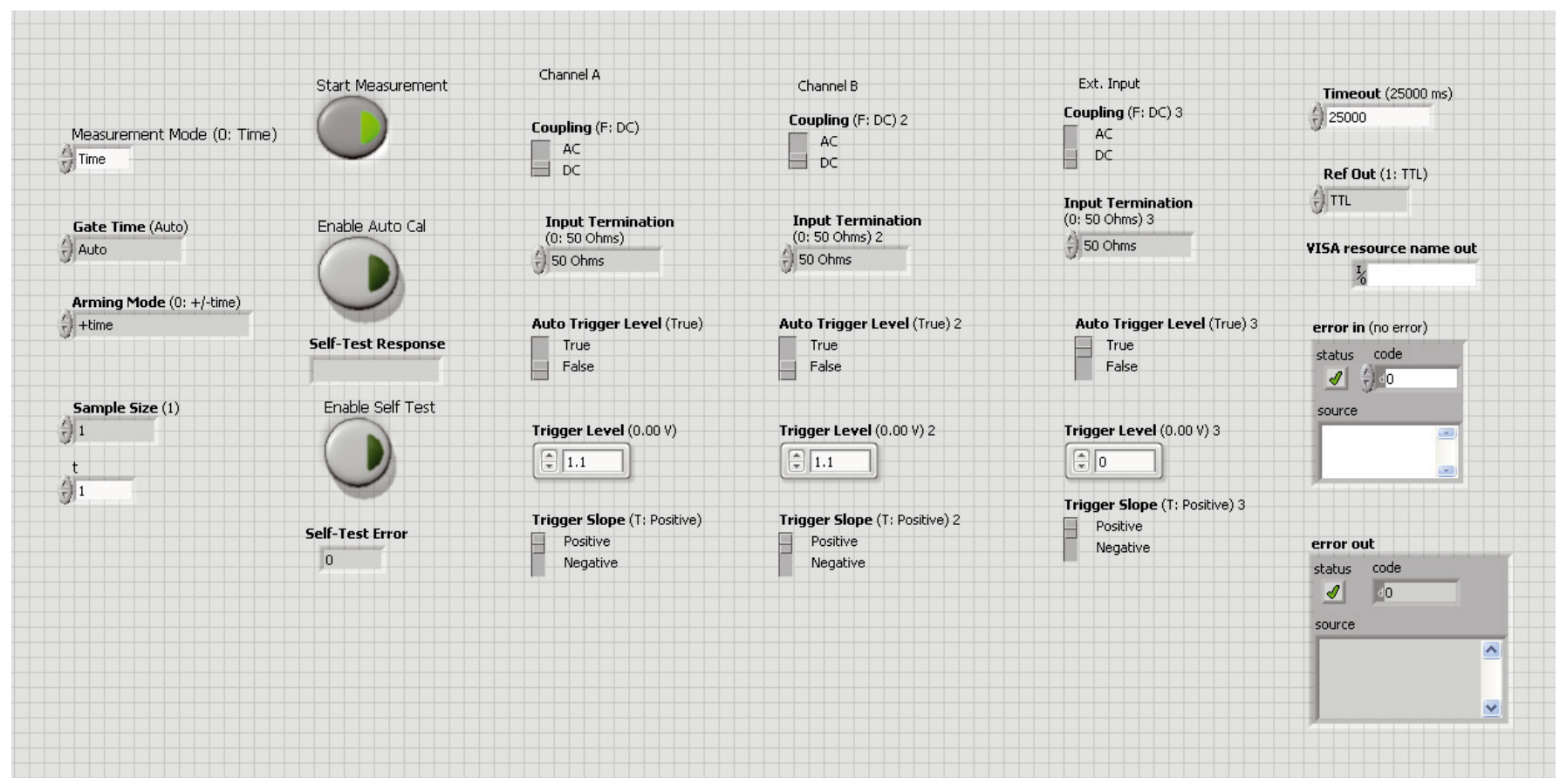

Fig. 8. The software front panel of the TIC.

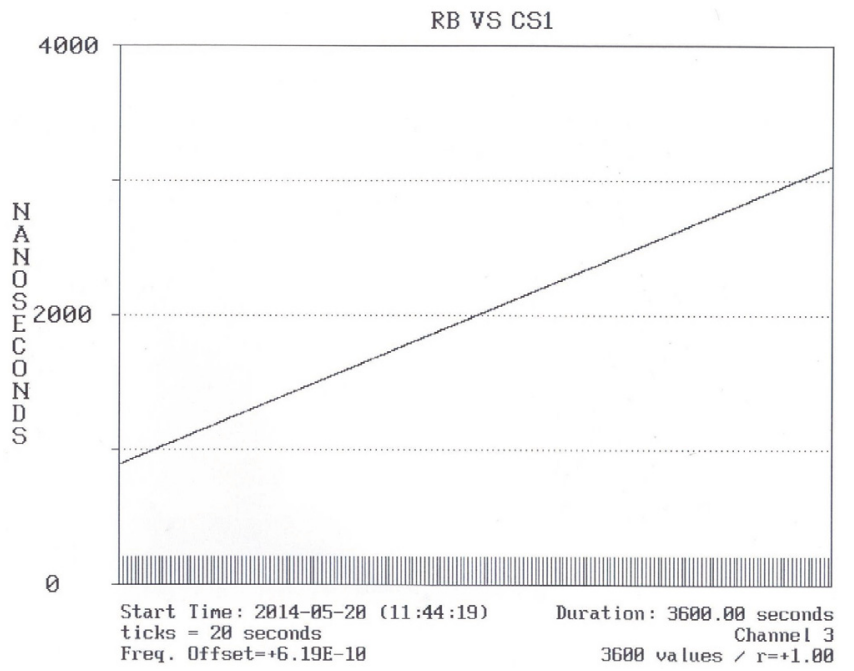

(a) phase difference plot generated by FMAS

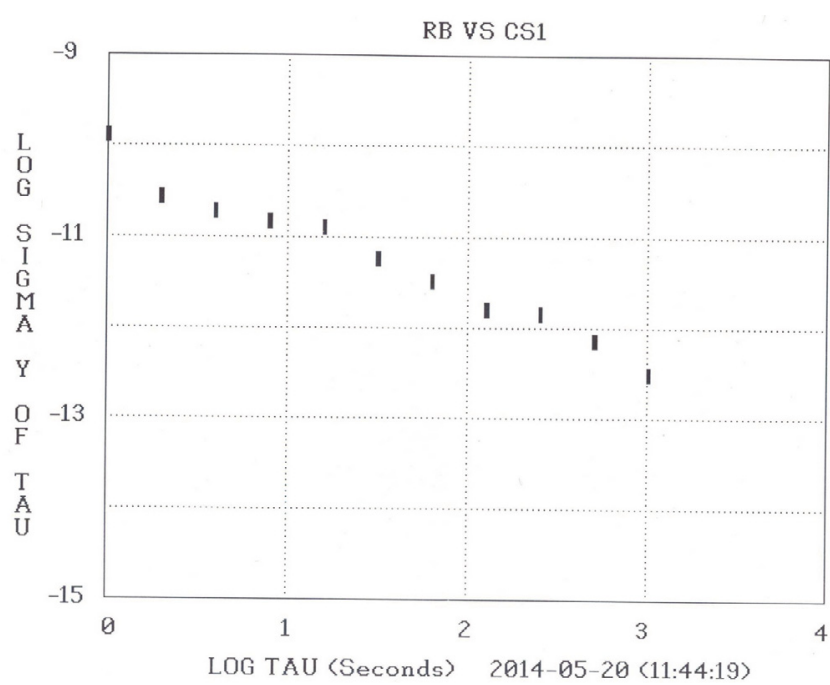

(b) Frequency Stability plot generated by FMAS

Fig. 9. The measurement results generated by the turn key solution.

from Symmetricom). The Rb oscillator was connected to both the FMAS and the GS at the same time. First, we measured the frequency offset and frequency stability of the oscillator using the FMAS. Then, we measured the same oscillator using the GS twice. One time using the PCM and the other time using the DFMM. Second, to verify the capability of the GS on measuring a frequency offset worse than $1 \times 10^{-5} \mathrm{~Hz} / \mathrm{Hz}$, we measured the frequency offset of a Cesium based frequency signal after adding to it an offset greater than $1 \times 10^{-5} \mathrm{~Hz} / \mathrm{Hz}$. In the following it will be shown in details the verification results.

\subsection{Calibrating a Rb frequency standard using the PCM}

The DUT is a $10 \mathrm{MHz}$ Rb frequency source that was calibrated versus the national primary frequency standard; which is a Cs frequency standard that has an accuracy of $5 \times 10^{-13} \mathrm{~Hz} / \mathrm{Hz}$.

The frequency offset and frequency stability for a measurement period of $1 \mathrm{~h}$ was measured using the FMAS and the GS. Figures $9 \mathrm{a}$ and $9 \mathrm{~b}$ show the phase difference plot and the frequency stability plot that were generated by the FMAS while Figures 10a and 10b show 
Table 1. Comparison between the stability results generated by the American and the Egyptian systems.

\begin{tabular}{ccc}
\hline $\begin{array}{c}\text { Averaging period (Tau) } \\
(\mathrm{s})\end{array}$ & $\begin{array}{c}\text { Turnkey system (FMAS) } \\
(\mathrm{Hz} / \mathrm{Hz})\end{array}$ & $\begin{array}{c}\text { Generalized system }(\mathrm{GS}) \\
(\mathrm{Hz} / \mathrm{Hz})\end{array}$ \\
\hline 1 & $1.3 \times 10^{-10}$ & $3.6 \times 10^{-11}$ \\
2 & $3 \times 10^{-11}$ & $2.5 \times 10^{-11}$ \\
4 & $2 \times 10^{-11}$ & $1.6 \times 10^{-11}$ \\
8 & $1.5 \times 10^{-11}$ & $8.5 \times 10^{-12}$ \\
16 & $1 \times 10^{-11}$ & $5.1 \times 10^{-12}$ \\
32 & $9 \times 10^{-12}$ & $2.7 \times 10^{-12}$ \\
64 & $3 \times 10^{-12}$ & $1.6 \times 10^{-12}$ \\
128 & $1.5 \times 10^{-12}$ & $1.5 \times 10^{-12}$ \\
256 & $1.3 \times 10^{12}$ & $8.5 \times 10^{-13}$ \\
512 & $9 \times 10^{-13}$ & $7.4 \times 10^{-13}$ \\
\hline
\end{tabular}
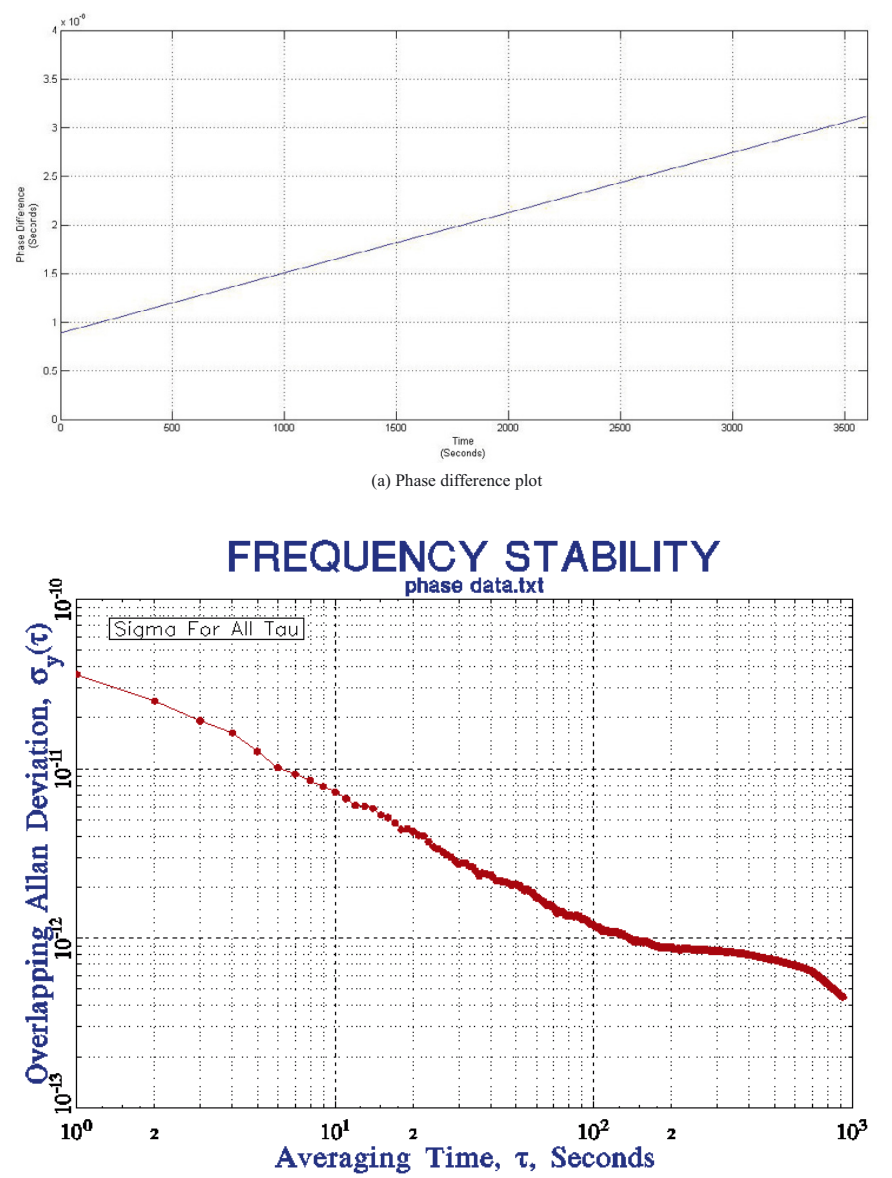

(b) Frequency Stability plot generated for all Tau

Fig. 10. The calibration results generated by the GS.

the phase difference plot and the frequency stability plot that were resulted from the GS. It is shown that the RFO that was measured by the turn key solution was $6.19 \times 10^{-10} \mathrm{~Hz} / \mathrm{Hz}$, while the value measured by the GS was $6.185 \times 10^{-10} \mathrm{~Hz} / \mathrm{Hz} \approx 6.19 \times 10^{-10} \mathrm{~Hz} / \mathrm{Hz}$; which is the same value. Table 1 summarizes the stability results.

From Table 1, it is shown that the GS generates almost the same stability results of the turn key solution. Moreover, we can get a stability plot for all the possible

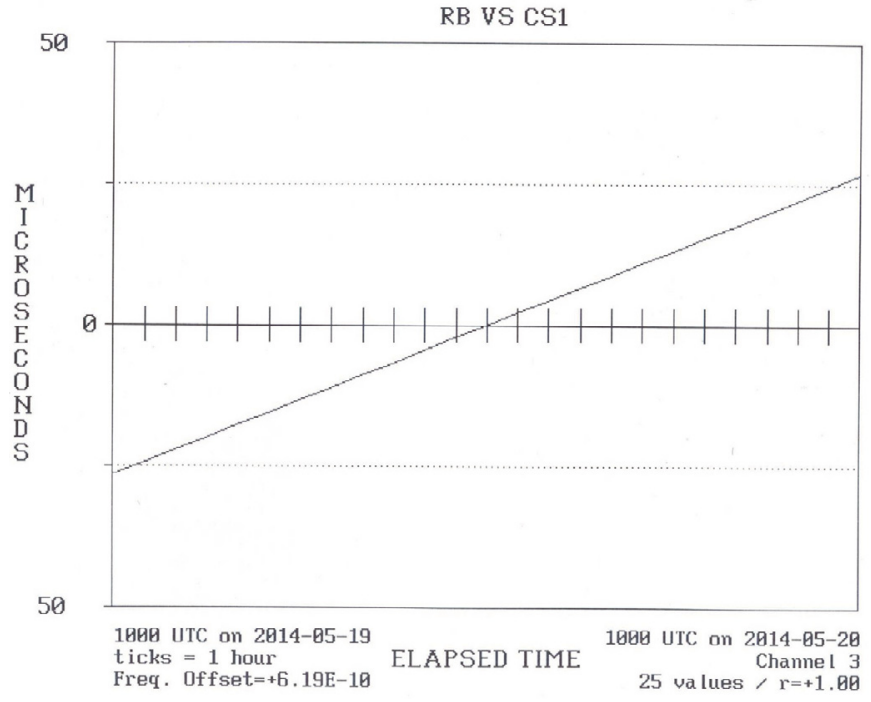

Fig. 11. The phase difference plot generated by FMAS.

averaging periods (Tau), like that is shown in Figure 10b; this option doesn't exist in the turn key system. This is because we can access the raw collected data and use other external software for extra analysis.

In general, when we calibrate a frequency standard versus a transfer standard (like GPS OR GLONASS) a measurement period of at least $24 \mathrm{~h}$ is normally used. FMAS has the capability of doing calibrations for a measurement period of $24 \mathrm{~h}$. After this period it prints a calibration sheet showing the phase difference relation between the DUT and the reference and the value of the RFO. Unfortunately, FMAS can't measure the frequency stability for a measurement period of $24 \mathrm{~h}$ but the GS can.

Figure 11 shows the phase difference plot and the RFO value that was generated from the FMAS when calibrating the same $\mathrm{Rb}$ oscillator for a measurement period of $24 \mathrm{~h}$. Figures $12 \mathrm{a}$ and $12 \mathrm{~b}$ show the phase difference plot and the frequency stability plot that were resulted from the GS. The value of the RFO generated by the FMAS and the GS are equal; which is $6.19 \times 10^{-10} \mathrm{~Hz} / \mathrm{Hz}$.

To verify the frequency stability plot generated by the GS for a measurement period of $24 \mathrm{~h}$ we can compare the first part of it that corresponds to a measurement period 


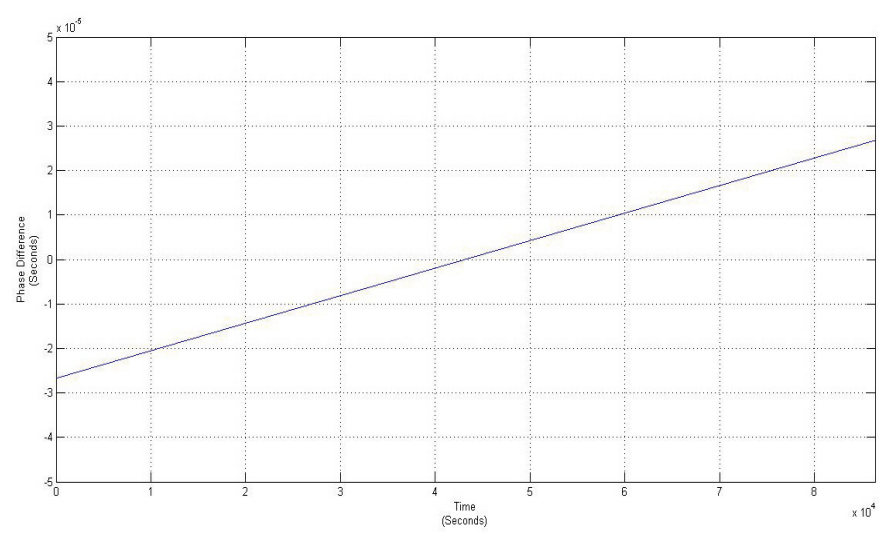

(a) The phase difference plot

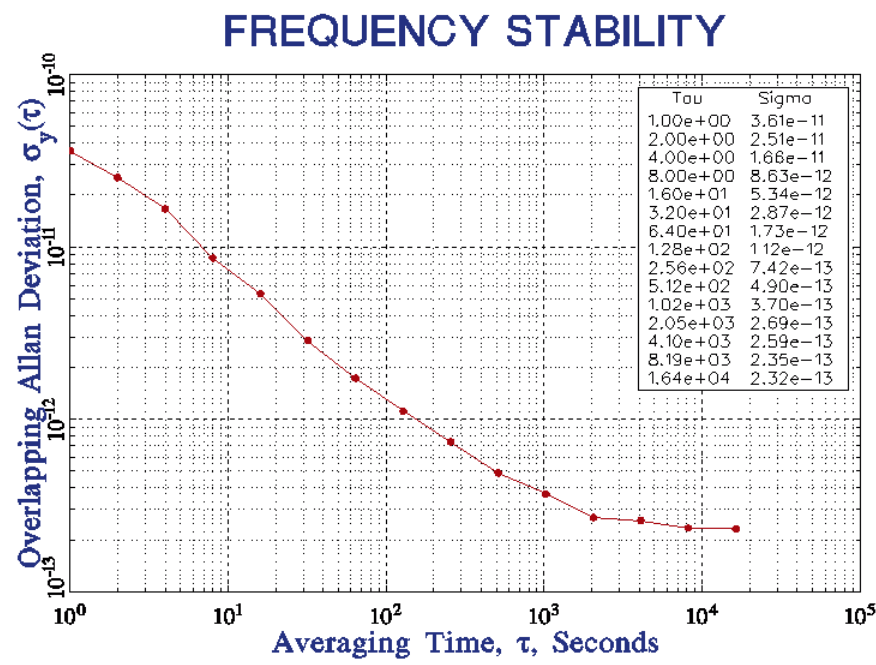

(b) The frequency stability plot generated by the CS

Fig. 12. The calibration plots generated by the GS.

of $1 \mathrm{~h}$ to the stability plot generated by the FMAS at the same measurement period, shown in Figure 9b. It is shown that they are almost equal.

\subsection{Measuring frequency sources having a RFO worse than $1 \times 10^{-5} \mathrm{~Hz} / \mathrm{Hz}$ using the PCMM}

According to the FMAS specifications, it can only measure a RFO smaller than $1 \times 10^{-5} \mathrm{~Hz} / \mathrm{Hz}$ and greater than $1 \times 10^{-13} \mathrm{~Hz} / \mathrm{Hz}$. This means that this system is not suitable for calibrating the commercial frequency sources that has a RFO greater than $1 \times 10^{-5} \mathrm{~Hz} / \mathrm{Hz}$. The GS offers this option. To prove that, we added a frequency offset of $1 \times 10^{-4} \mathrm{~Hz} / \mathrm{Hz}$ (using an Arbitrary Function Generator, Agilent AFG3252) to a Cs-based $10 \mathrm{MHz}$ sinusoidal frequency signal. We calibrated this frequency versus the same primary frequency standard using both the FMAS and the GS. Since the RFO of this signal is outside the specifications of the FMAS, the displayed readings of its internal counter was unstable. On the other hand, Figure 13 shows the phase difference plot and the RFO plot that were resulted from the GS. It is shown that the measured RFO by the GS was $9.99 \times 10^{-5} \mathrm{~Hz} / \mathrm{Hz}$.

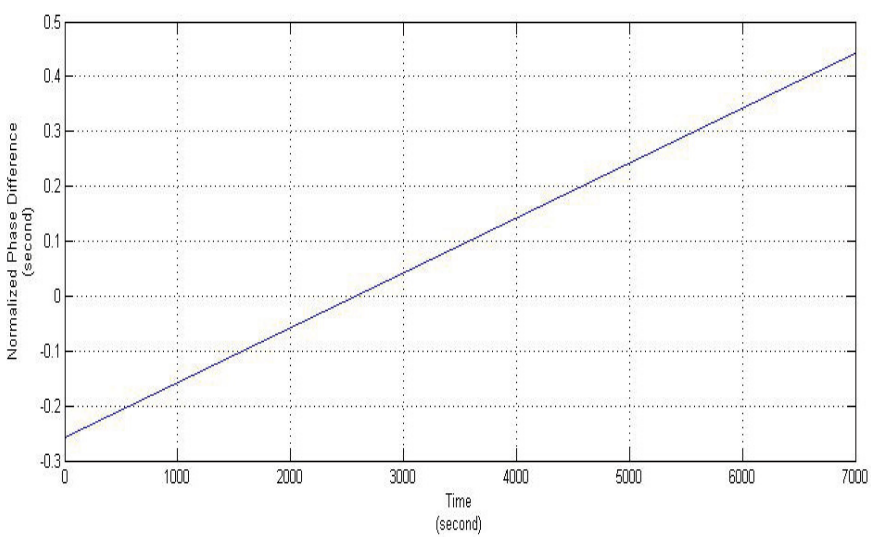

(a) The phase difference plot

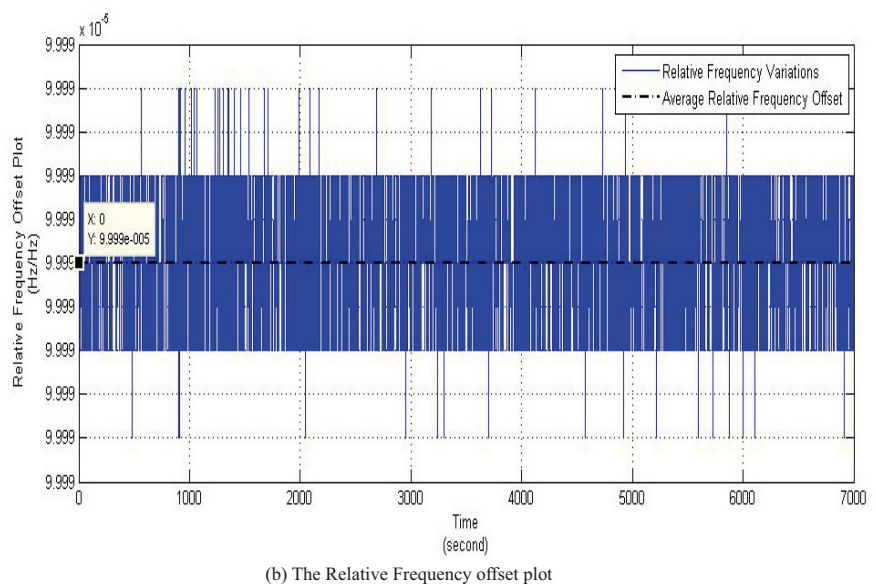

Fig. 13. The calibration plots generated by the GS.

\subsection{Calibration using the DFMM}

At the time the FMAS can only use the PCM in calibrating/monitoring frequency sources, the GS can use both the PCM and the DFMM in the calibration process.

To verify the results generated by the GS using the DFMM we calibrated the same Rb frequency source that has been calibrated before using the PCM. Figures 14a and $14 \mathrm{~b}$ show the phase difference plot and the stability plot that were resulted from the FMAS. It is shown that the RFO is $6.20 \times 10^{-10} \mathrm{~Hz} / \mathrm{Hz}$. For the GS, Figures $15 \mathrm{a}$ and $15 \mathrm{~b}$ show the RFO and stability plots. It is shown that the measured average RFO was $6.87 \times 10^{-10} \mathrm{~Hz} / \mathrm{Hz}$. The drift from the value calculated by the FMAS is due to a drift in the accuracy of the counter in the frequency mode that has to be taken into consideration. So this drift is measured by measuring the frequency of the counter's time base by the counter itself. Figure 16 shows that there is a drift in the frequency measurement accuracy of the counter by about $6.19 \times 10^{-11} \mathrm{~Hz} / \mathrm{Hz}$. This value has to be subtracted from the value generated by the GS to be $6.25 \times 10^{-10} \mathrm{~Hz} / \mathrm{Hz}\left(6.87 \times 10^{-10}-6.19 \times 10^{-11}\right)$; which is very close to the one generated by the FMAS. In this case, it was expected to have a small drift between the two measurement systems because they use two different measurement methods. 


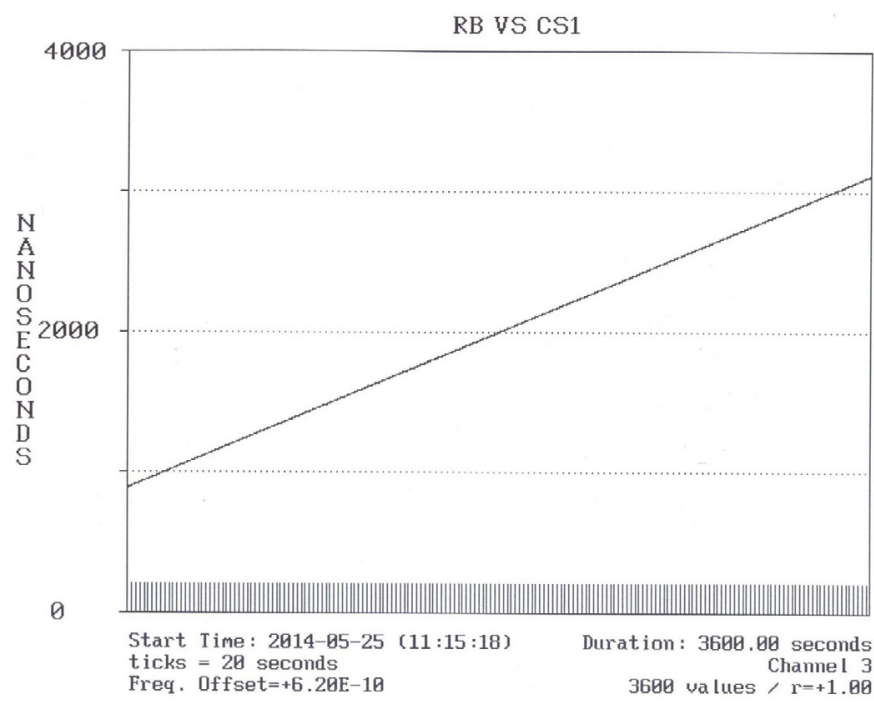

(a) the phase difference plot generated from FMAS

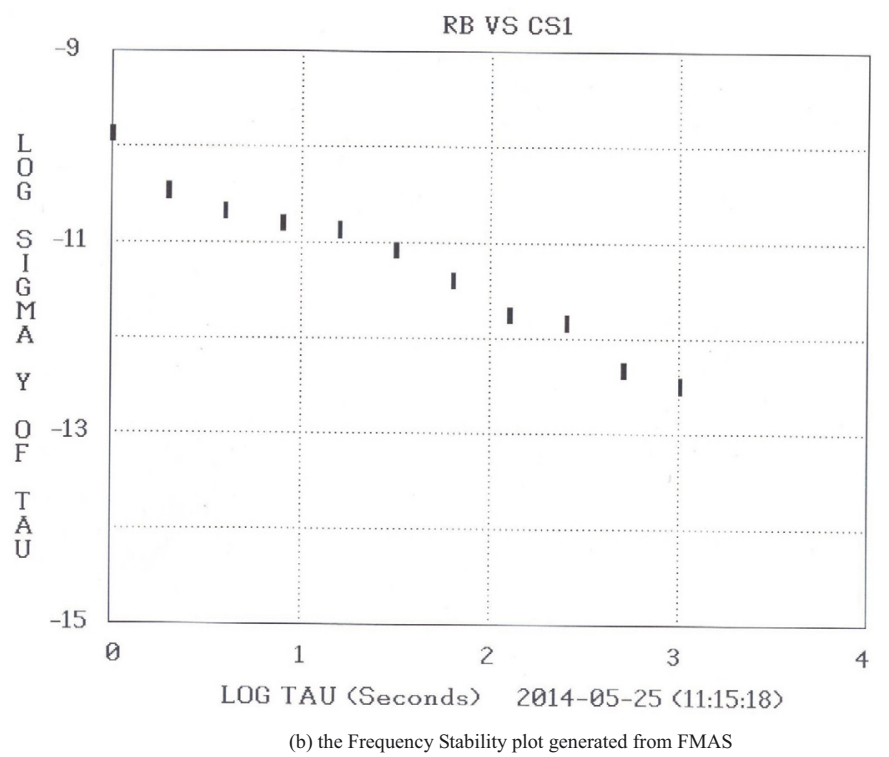

Fig. 14. The calibration plots generated by the FMAS.

\section{Conclusion}

Any time and frequency laboratory either it was a calibration lab or a national metrology lab should maintain at least two automatic systems for frequency measurements. The first one is dedicated for monitoring the performance of the primary frequency standards inside the lab. That is why this system should use a high accuracy measurement method; which is the PCM. The second system is dedicated for calibrating the general or less accurate frequency sources coming from the industry. This system can use a less accurate measurement method; which is the DFMM. Moreover, sometimes the time and frequency calibration lab are asked for calibrating precise frequency sources. So they either have to maintain a third measurement system that is dedicated for calibrating such precise frequency sources or use multichannel measurement systems that

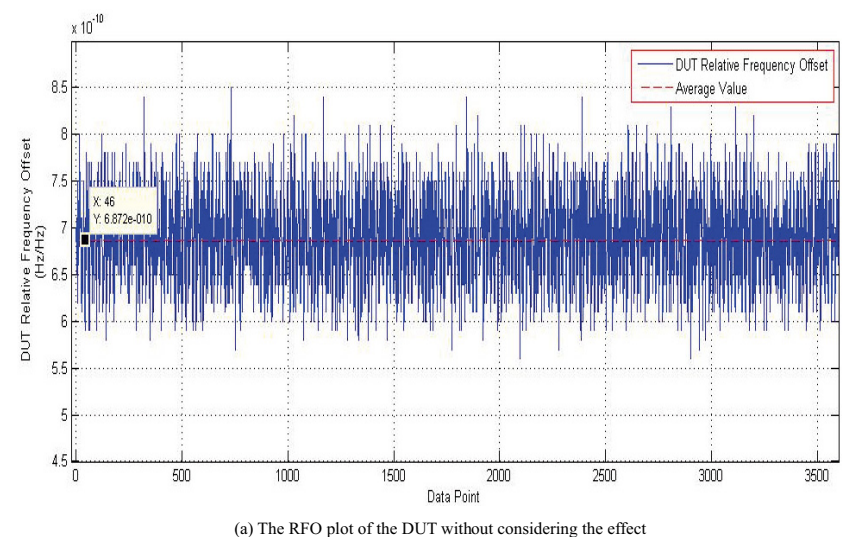

FREQUENCY STABILITY

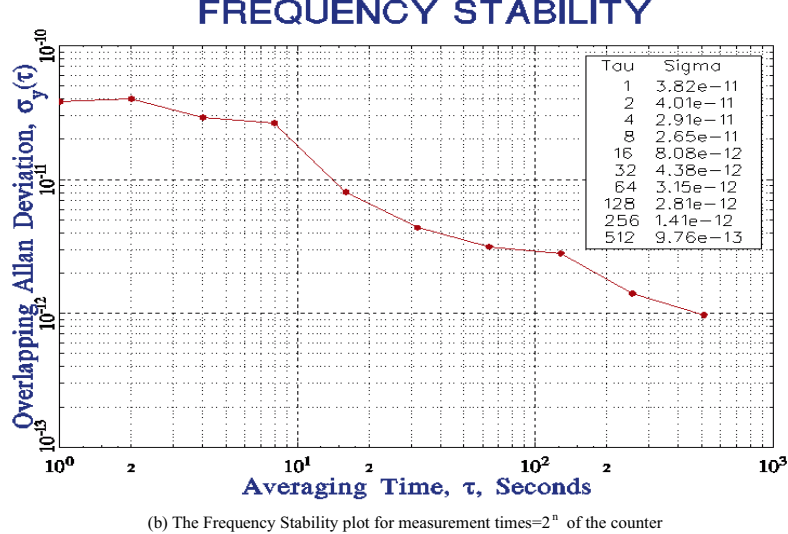

Fig. 15. The calibration plots of the GS.

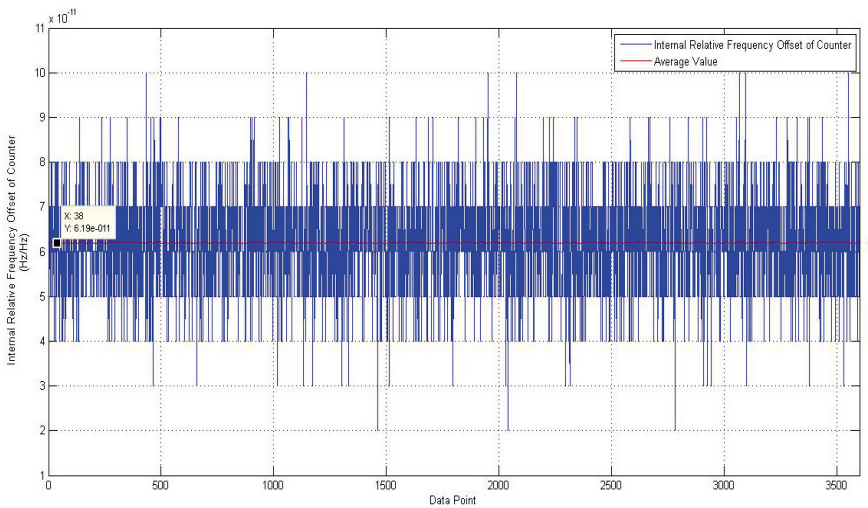

Fig. 16. The drift in the accuracy of frequency measurement by the counter.

can both monitor the primary standard of the lab and at the same time calibrate the precise frequency sources coming from outside. In this paper an automated, a multichannel, and a Generalized System for monitoring and calibrating the primary, secondary, and commercial frequency sources was introduced. This GS can be easily maintained and upgraded to follow both the customer's needs and the continuous development in the field of electronics. The operation of that system was verified through comparing its results to the results generated by one of the turnkey systems. 
Acknowledgements. The authors acknowledge the Science and Technology Development Fund (STDF) for financing purchasing the equipments that have been used in the measurement setup.

\section{References}

1. M.A. Lombardi, Fundamentals of Time and Frequency (NIST Publication, CRC Press, LLC, 2002)

2. D.A. Howe, D.W. Allan, J.A. Barnes, Properties of Signal Sources and Measurement Methods, Proceedings of the 35th Annual Symposium on Frequency Control (1981)

3. J. Jespersen, Introduction to the Time Domain Characterization of Frequency Standards. Proc. 23th Annu. Precise Time and Time Interval (PTTI) Meeting (Pasadena, CA. 83-102, 1991)

4. D.A. Howe, D.W. Allan, J.A. Barnes, Properties of Signal Sources and Measurement Methods, Proceedings of the 35th Annual Symposium on Frequency Control (1981)

5. J. Kalisz, Review of methods for time interval measurements with picosecond resolution, Metrologia 41, 17-32 (2004)

6. J.T. Stanley, J.B. Milton, Basic Laboratory Methods for Measurement or Comparison of Frequencies and Time Intervals, NBS REPORT, U.S. Department of Commerce, National Bureau of Standards, Institute for Basic Standards, Boulder Colorado, 80302 (1972)

7. M.A. Lombardi, National Institute of Standards and Technology (NIST), Boulder, Colorado, Legal and Technical Measurement Requirements for Time and Frequency, Review Paper, NIST Publication
8. J. Kalisz, Review of methods for time interval measurements with picosecond resolution, Metrologia 41, 17-32 (2004)

9. Understanding frequency counter specifications, Application Note 200-4, Electronic Counters Series, Hewlett Packard, USA

10. More hints for making better frequency counter measurements, Application Note 1499, Agilent Technologies, USA

11. Fundamentals of the electronic counters, Application Note 200, Electronic Counter Series, Agilent Technologies, USA

12. Application Note, Hints for Getting the Most from Your Frequency Counter, Agilent Technologies

13. SR620 universal time interval counter, user manual, Revision 2.7 (2/2006), Stanford Research Systems

14. G.G. Hamza, Investigation of the Optimum Trigger Level in Time Interval Measurement, MAPAN-Journal of Metrology Society of India (2014)

15. Hewlett-Packard Company, Fundamentals of Time Interval Measurement, Hewlett-Packard Application Note 200-3, 5965-7663E (1997) 68

16. Sources of error in time interval measurements, Application Note, Fluke Corporation, USA

17. http://www.spectradynamics.com/product-sheets/ PPS-2RM-B1_2010.pdf, November 2014

18. Bureau International des Poids et Mesures, The International System of Units (SI), 8th edn., Organisation Intergouvernementale de la Convention du Me'tre (2006)

19. G. Hamza, A. Zekry, I. Motawie, Implementation of a complete GPS receiver using simulink, IEEE circuits and systems magazine, Fourth Quarter (2009) 